\title{
Laser Melting of Nanoparticulate Transparent Conductive Oxide Thin Films
}

\author{
Marcus BAUM $^{* 1,2}$, Sebastian POLSTER ${ }^{* 3}$, Michael P.M. JANK ${ }^{* 4}$, Ilya ALEXEEV ${ }^{* 1,2}$, Lothar FREY ${ }^{* 3,4}$ \\ and Michael SCHMIDT $^{* 1,2}$ \\ ${ }^{* 1}$ Chair of Photonic Technologies, Friedrich-Alexander-University Erlangen-Nuremberg, Paul-Gordan-Str. 3, 91052 \\ Erlangen, Germany \\ E-mail:marcus.baum@lpt.uni-erlangen.de \\ ${ }^{* 2}$ Erlangen Graduate School in Advanced Optical Technologies (SAOT), Friedrich-Alexander-University Erlangen- \\ Nuremberg, Am Weichselgarten 8, 91058 Erlangen, Germany \\ ${ }^{* 3}$ Chair of Electron Devices, Friedrich-Alexander-University Erlangen-Nuremberg, Cauerstr. 6, 91058 Erlangen, Germany \\ ${ }^{* 4}$ Fraunhofer Institute for Integrated Systems and Device Technology, Schottkystr. 10, 91058 Erlangen, Germany
}

Layers of $\mathrm{ZnO}$ nanoparticles with thicknesses of about $40 \mathrm{~nm}$ were prepared on silicon substrates. The layers were irradiated by single pulses of a $248 \mathrm{~nm}$ excimer laser, which proofed suitable for consolidation and significant densification of the particle layers under ambient conditions. Experiments as well as simulations have confirmed that the application of a $\mathrm{SiO}_{2}$ particle layer between the substrate and the $\mathrm{ZnO}$ particle layer can be used to hamper heat transfer towards the substrate. Thus the $\mathrm{ZnO}$ layer can be thermally insulated from the substrate while heating the $\mathrm{ZnO}$ up to its extremely high melting point. Consequently, such a layer stack could enable the application of consolidated particle layers on temperature-sensitive carrier substrates such as polymer foils which are to be used in low-cost mass production of devices like displays or solar cells.

DOI:10.2961/jlmn.2013.02.0005

Keywords: $\mathrm{ZnO}$, nanoparticles, laser melting, transparent, conducting layers

\section{Introduction}

Transparent conductive oxides, such as tin-doped indium oxide (ITO) or doped $\mathrm{ZnO}$, exhibit a high electrical conductivity while at the same time offering transparency in the visible spectral range. Due to these properties they are suitable for a wide range of applications in optoelectronic devices, such as LC-displays, touchscreens or solar cells [1]. In most commercial products, the required conducting films are created by vapor deposition. However, for the low cost mass production of such devices, avoidance of the costly vacuum deposition step would be beneficial. This can be achieved by solution processing of the material by various printing techniques. A common method is the deposition of thin films out of nanoparticle dispersions [2, 3]. Usually the layer properties, e.g. its electrical conductivity can be enhanced by a subsequent annealing step, which can induce melting or sintering of the particles. By such a consolidating treatment the charge carrier transport through the film is greatly facilitated by enlargement of the particle-particle interfaces [4,5]. However, if low cost substrates like polymer foils are to be used, the processing temperatures need to be kept low in order to protect these substrates from thermal damage. To overcome this problem, we propose UV laser treatment to restrict energy deposition to the surface of the particle layer [6]. Furthermore, we show both by experiments and simulations that a layer of $\mathrm{SiO} 2$ particles sandwiched between the substrate and the $\mathrm{ZnO}$ particle layer can be used as a thermally insulating buffer layer which prevents heat transfer towards the substrate and thus also contributes to the protection of the substrate. We chose zinc oxide nanoparticles as an exemplary metal oxide nanoparticle system since it is a well-studied semiconducting material [7] which can be deposited from a solution via nanoparticle dispersions.

\section{Simulation}

To simulate the temperature distribution and the temperature course within a nanoparticle layer during and after laser treatment, finite element simulations in COMSOL 3.5 were carried out. The effect of a $\mathrm{SiO}_{2}$ buffer layer as well as the influence of different parameters such as pulse length and the gas surrounding the particle layer were investigated.

Stacks of a $40 \mathrm{~nm}$ thick $\mathrm{ZnO}$ particle layer consisting of $\mathrm{ZnO}$ particles with a diameter of about $25 \mathrm{~nm}$ followed by a buffer layer of a $220 \mathrm{~nm}$ thick $\mathrm{SiO} 2$ layer consisting of $\mathrm{SiO} 2$ particles with a diameter of $50 \mathrm{~nm}$ on a Si substrate (from now on referred to as "stack A") and of a $40 \mathrm{~nm}$ thick $\mathrm{ZnO}$ particle layer with a particle diameter of $25 \mathrm{~nm}$ on a Si substrate ("stack B") were considered in the simulation. The described model setup can be seen in Fig 1. The particular values for the layer thicknesses and particle sizes were taken to match the experimental part. For the energy input single pulses of a $248 \mathrm{~nm}$ Excimer laser with a pulse length of $30 \mathrm{~ns}$ were used. The wavelength of $248 \mathrm{~nm}$ cor- 
responds to a photon energy of $5 \mathrm{eV}$ which exceeds the band gap of $\mathrm{ZnO}$ of about $3.4 \mathrm{eV}$ [7]. Thus, it is very well absorbed in $\mathrm{ZnO}$ due to interband transitions leading to a very shallow penetration depth of about $50 \mathrm{~nm}$ in bulk $\mathrm{ZnO}$ [8]. In the simulations the laser pulse was modeled as rectangular in time, which is in good agreement with its actual temporal shape. Absorption of laser light in $\mathrm{Si}$ and $\mathrm{ZnO}$ was calculated according to Beer-Lambert law. Reflection of the laser light at boundaries of different materials was estimated by the Fresnel equations, while the optical properties of the different particle layers were averaged according to Bruggemann's model [9]. It should be mentioned here that the absorbed laser energy is assumed to be an ideal heat source neglecting all other effects like radiative transfers. The laser fluence was set to $245 \mathrm{~mJ} / \mathrm{cm}^{2}$ which is the fluence needed to achieve good $\mathrm{ZnO}$ particle consolidation in the samples without the $\mathrm{SiO}_{2}$ buffer layer (see experimental part, Fig. 6 d). The dissipation of heat in the particle layer was calculated by the heat transfer equation, also thermal radiation of the surfaces was taken into account. The temperature-dependent bulk thermal properties of $\mathrm{ZnO}, \mathrm{SiO}_{2}, \mathrm{Si}$ and air as well as the optical properties of $\mathrm{ZnO}$ and $\mathrm{Si}$ were taken from the literature [8, 10-14]. An appropriate heat sink for the incident laser energy was integrated by extending the lateral dimensions of the air side and the Si substrate side of the simulation area to $10 \mu \mathrm{m}$ and $100 \mu \mathrm{m}$, respectively, resulting in constant temperatures at the limits of the simulation area during the whole simulated time frame of $1 \mu \mathrm{s}$. The transverse boundaries of the $150 \mathrm{~nm}$ wide simulation area were assumed to be thermally insulating resulting in periodic boundary conditions which should be reasonable as the laser spot is wider by several orders of magnitude. The calculation with the above parameters results in maximum temperatures of about $1200{ }^{\circ} \mathrm{C}$ for stack B, a temperature much lower than the melting point of $\mathrm{ZnO}$ of about $1975{ }^{\circ} \mathrm{C}$ [15]. Thus, according to the simulation the laser pulse would not have led to particle melting and consolidation. The reason for this underestimation of temperature is that the thermal conductivity of the used material was not represented correctly by the values of thermal conductivity for the bulk material. Since the used particles are only a few tens of nanometers in diameter, thermal conductivity i.e. phonon spread in the material is significantly reduced as the spatial dimension of the phonons is comparable to the particle size and the contact area between the particles. Consequently, this was taken into account by applying thermal conductivities measured on a comparable material [16]. This means that the

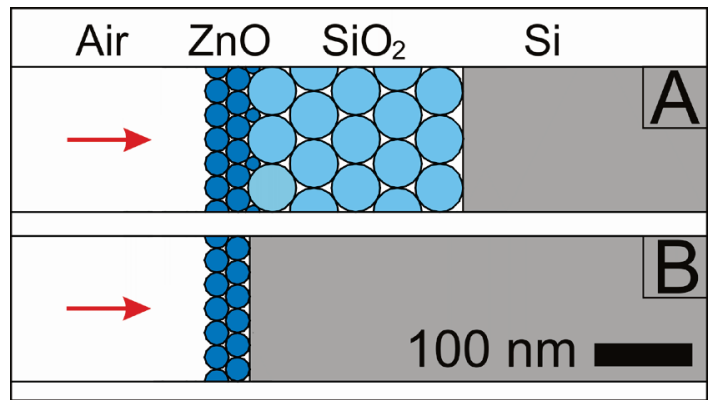

Fig. 1 Model setup used in the simulation (compare Fig. 6 (a) and Fig. 7(a)). The red arrows indicate the direction of the incident laser pulse
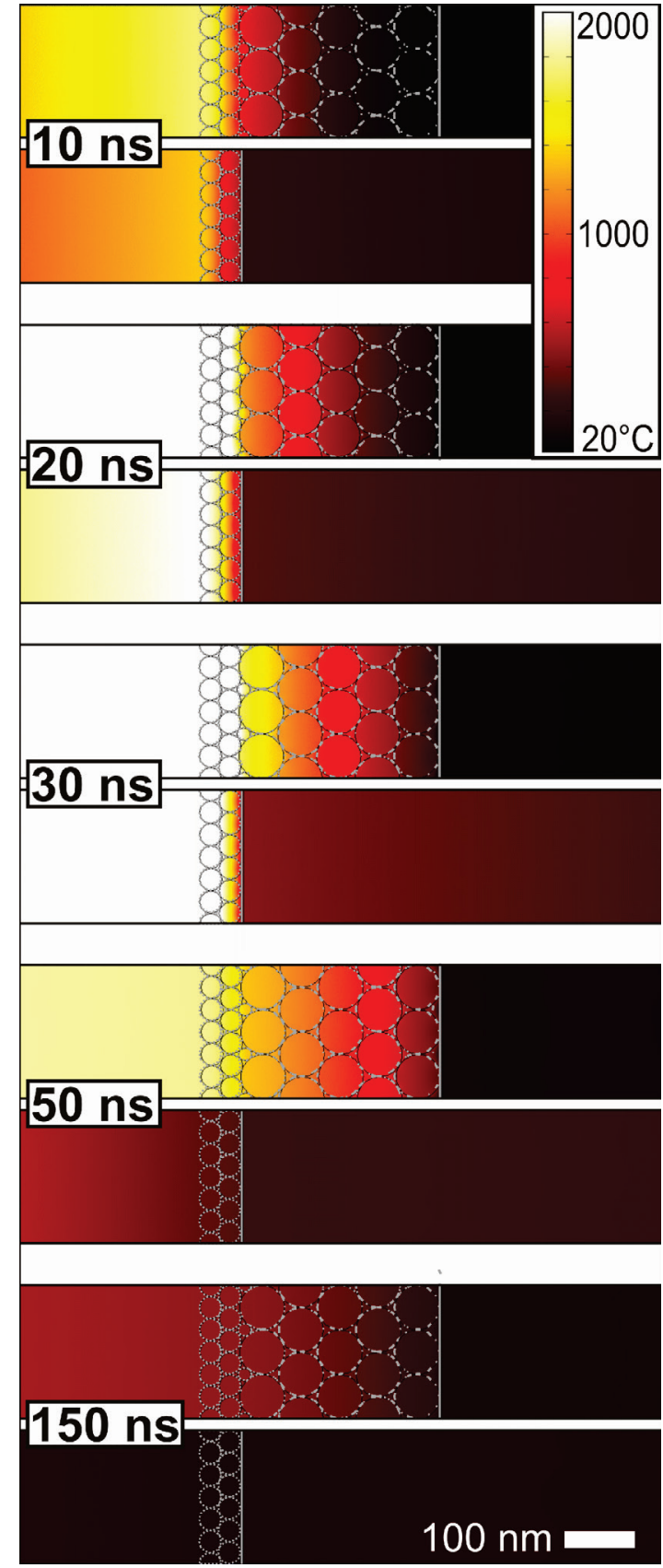

Fig. 2 Temperature distributions in the particle layers (see Fig. 1) at five exemplary time steps. Room temperature appears in black, while for clarity temperatures of $2000{ }^{\circ} \mathrm{C}$ and above are shown in white.

special properties of the material due to its nanoscale size are changed in our favor. Smaller particles should reduce the thermal conductivity even further while at the same time lowering the melting point [17] resulting in a better compatibility with sensitive substrates. The results of the simulations for five representative time steps are visualized in in Fig. 2. It can be seen that both particle systems reach temperatures of $2000{ }^{\circ} \mathrm{C}$ or more in the $\mathrm{ZnO}$ layer by the end of the incident $30 \mathrm{~ns}$ laser pulse. While for stack B i.e. a $\mathrm{ZnO}$ particle layer without underlying buffer layer, the substrate is substantially heated up, for stack A the substrate stays comparatively cool under the $\mathrm{SiO}_{2}$ buffer layer. The color gradient in the buffer layer shows that heat transfer towards the substrate is hampered by the $\mathrm{SiO}_{2}$ particle layer. It also becomes clear that the $\mathrm{ZnO}$ layer cools down 

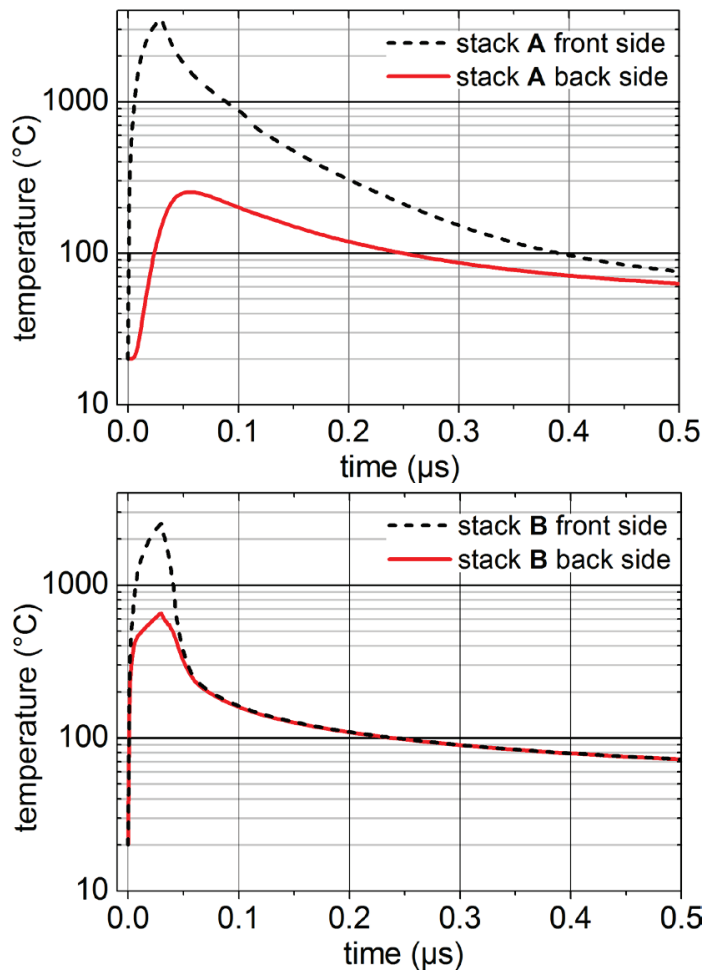

Fig. 3 Temperature course at the interface between air and the $\mathrm{ZnO}$ particle layer ("front side") and at the interface between the particle layer stack and the substrate ("back side") after irradiation with a $30 \mathrm{~ns}$ laser pulse beginning at $\mathrm{t}=0 \mathrm{~s}$ with a fluence of 245 $\mathrm{mJ} / \mathrm{cm}^{2}$

more slowly for the layer stack with underlying buffer layer. A more quantitative representation of the outcome of the simulation is shown in fig. 3. It shows that particle stack B reaches a maximum temperature of about $2500{ }^{\circ} \mathrm{C}$, a temperature well above the melting temperature of $\mathrm{ZnO}$, while the maximum temperature of stack $\mathrm{A}$ is about $1000{ }^{\circ} \mathrm{C}$ higher which is a consequence of neglecting phase changes of the particle layer in the simulation. The cooling phase of the layer surface turns out to be significantly longer for stack A. The temperature right at the interface between the particle layer and the substrate is above $650{ }^{\circ} \mathrm{C}$ and shows steep gradients for stack B, while it stays below $250{ }^{\circ} \mathrm{C}$ and displays much lower gradients both in the heating and in the cooling phase for stack A. This outcome indicates that the same kind of buffer layer can be used to protect more sensitive substrates such as polymer foils which are required for the low-budget mass production of electron devices. It should be emphasized that the laser fluences needed for consolidation of the particle layer are reduced by applying a buffer layer and that a thicker buffer layer decreases the temperatures reached on the substrate side even more. The influence of the $\mathrm{SiO}_{2}$ buffer layer thickness is shown in Fig. 4. The temperature course at the interface between the particle layer and the Si substrate was investigated in further simulations for different $\mathrm{SiO}_{2}$ layer thicknesses (90 nm, $220 \mathrm{~nm}$ and $350 \mathrm{~nm})$. It clearly shows that the maximum temperature reached at this interface is highly dependent on the buffer layer thickness. Thus, this layer should be tuned in thickness depending on the substrate material and maintained at a thickness high enough to guarantee substrate protection but at the same time low enough to not diminish the visual quality e.g. in display

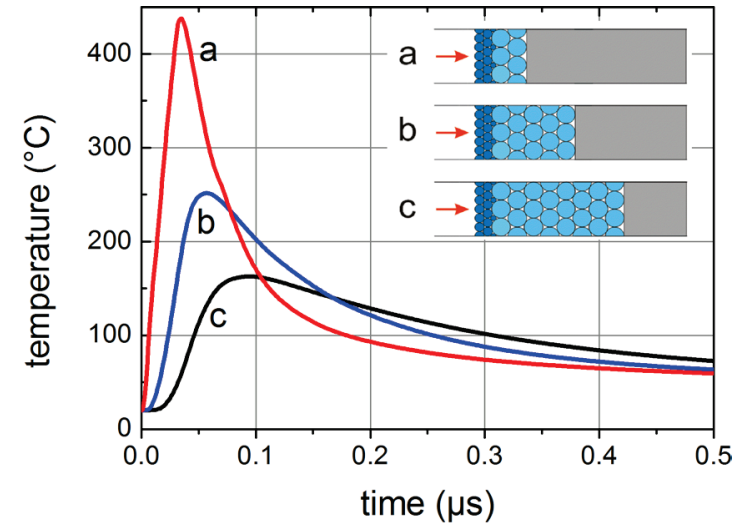

Fig. 4 Temperature course at the boundary between the particle layers and the $\mathrm{Si}$ substrate for different $\mathrm{SiO}_{2}$ buffer thicknesses (a $93 \mathrm{~nm}$, b $220 \mathrm{~nm}$, c $350 \mathrm{~nm}$ ). The inset shows schemes of the corresponding simulated layer stacks

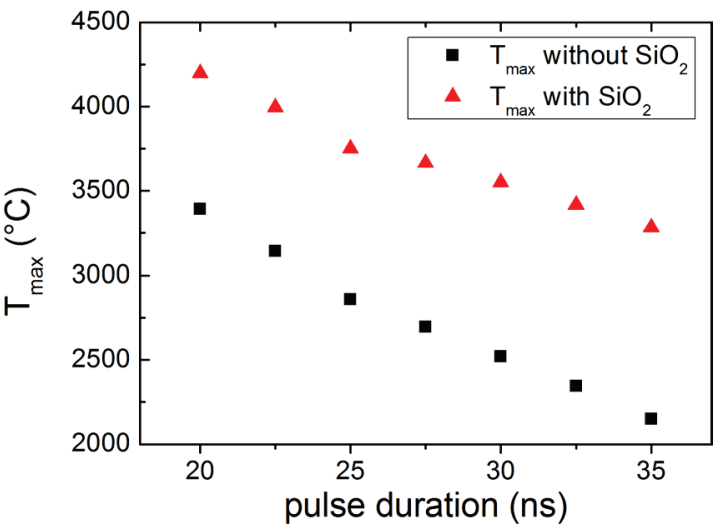

Fig. 5 Maximum temperatures reached at the boundary between air and the particle layer stack surface for particle layers with and without buffer layer for different pulse durations at a fluence of $245 \mathrm{~mJ} / \mathrm{cm}^{2}$ (stack A and B)

applications. The lasing gas mixture of excimer lasers such as the one we used ages over time due to the formation of chemical compounds of the highly reactive halogen gases. This leads to a decrease in pulse energy for a given excitation voltage as well as to a slight decrease of the pulse length on the order of $10 \mathrm{~ns}$ when the laser gas becomes older. This decrease in pulse energy can be compensated by increasing the excitation voltage. However, the pulse length remains shortened. While this fact is not such a big issue in the common fields of application of excimer lasers, such as lithography, it turned out to be a challenge when consolidated layers of unchanged quality shall be produced. Consequently, the influence of such a variation of the pulse length was also examined by the simulation model. The results for the maximum surface temperatures layers with and without a $220 \mathrm{~nm}$ thick $\mathrm{SiO}_{2}$ buffer are shown in Fig 5 . It can be seen that the maximum temperature reached at the layer surface varies by a few hundreds of degrees Celsius when the pulse length is changed by several nanoseconds. Thus the pulse length should be monitored during sample treatment. For layer production over several days, the process window will have to be adapted. Moreover, simulations with different gas atmospheres (Argon and Helium) were carried out. However, they showed that within the limits of the model i.e. only taking into account the thermal properties of the gases and neglecting other effects e.g. possible chemical reactions or changes in stoichiometry, 

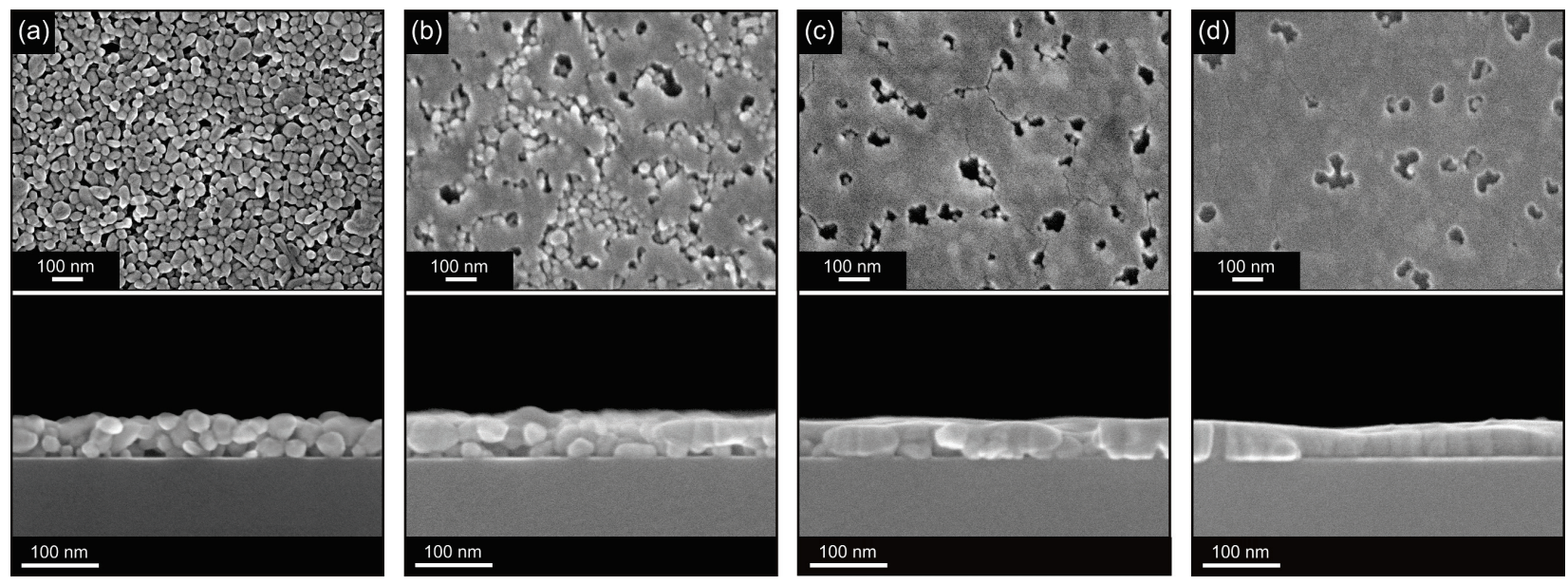

Fig. 6 Top-view and cross-sectional SEM images of different $40 \mathrm{~nm}$ thick $\mathrm{ZnO}$ particle layers on Si substrates. (a) untreated sample, (b) after irradiation by a single laser pulse at a fluence of $162 \mathrm{~mJ} / \mathrm{cm}^{2}$, (c) $187 \mathrm{~mJ} / \mathrm{cm}^{2}$, (d) $245 \mathrm{~mJ} / \mathrm{cm}^{2}$

the type of gas atmosphere does only marginally change the maximum temperatures reached.

\section{Experimental part}

In our experiments, we used $\mathrm{ZnO}$ nanoparticles commercially available from Evonik Industries AG, with a broad particle size distribution ranging from $20 \mathrm{~nm}$ to $120 \mathrm{~nm}$. An ethanol based dispersion was prepared from $20 \mathrm{wt} \%$ of these particles, while agglomeration of the particles was prevented by addition of $3 \mathrm{wt} \%$ 3,6,9-trioxadecanoic acid acting as a steric stabilizer. For the generation of a finer and more uniform particle size distribution, the suspensions were centrifuged resulting in an average particle size of about $25 \mathrm{~nm}$. The preparation of the suspension is described in detail in [18]. For the generation of thermally insulating buffer layers we used the commercially available suspension 30V50 from Klebosol containing $\mathrm{SiO}_{2}$ particles with an average particle diameter of about $50 \mathrm{~nm}$. The samples were prepared on p-type silicon wafer substrates with a dimension of $20 \mathrm{~mm} \times 20 \mathrm{~mm}$ and a thickness of
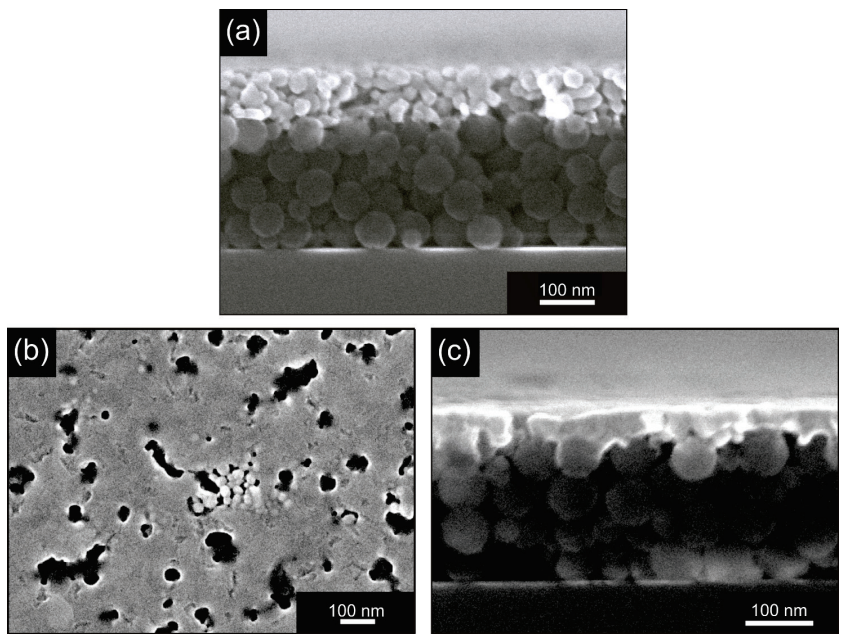

Fig. 7 (a) Cross-sectional SEM image of a $\mathrm{ZnO}$ particle layer (about $25 \mathrm{~nm}$ particle diameter) on a $220 \mathrm{~nm} \mathrm{SiO} 2$ particle layer on Si substrate. (b) Top-view and (c) cross-sectional SEM images of a layer stack consisting of a $40 \mathrm{~nm} \mathrm{ZnO}$ particle layer on a 220 $\mathrm{nm} \mathrm{SiO}$ layer after irradiation with a laser pulse at a fluence of 60 $\mathrm{mJ} / \mathrm{cm}^{2}$
$1 \mathrm{~mm}$. Prior to the deposition of the particle layers, the substrates were cleaned for $30 \mathrm{~s}$ in diluted HF (1\%) and then rinsed in deionized water. Layers of about $40 \mathrm{~nm}$ of $\mathrm{ZnO}$ particles or of about $220 \mathrm{~nm} \mathrm{SiO}_{2}$ particles followed by 40 $\mathrm{nm} \mathrm{ZnO}$ particles were generated by spin coating the, suspensions on the substrates (Fig 6 a, Fig 7 a). $\mathrm{SiO}_{2}$ particles were chosen for various reasons: they can be applied by the same technique as the $\mathrm{ZnO}$ particle layer, they do not significantly influence the optical appearance of the layer stack and feature a very low thermal conductivity. In order to thermally remove the stabilizing agents free of residues the samples were annealed at $400{ }^{\circ} \mathrm{C}$ for 30 min under ambient conditions. For the laser treatment of the particle layers, we used a Lambda Physik LPX 315i KrF excimer laser with a wavelength of $248 \mathrm{~nm}$, a raw beam size of $30 \mathrm{~mm}$ x $15 \mathrm{~mm}$, maximum pulse energy of $0.8 \mathrm{~J}$ and a pulse length of $30 \mathrm{~ns}$ while the temporal shape of the laser pulses is rectangular (we have to mention here that these values are measured for fresh laser gas. Obviously the maximum pulse energy decays over time, while the pulse length shortens slightly). The particle layers were irradiated by single pulses with fluences ranging from $60 \mathrm{~mJ} / \mathrm{cm}^{2}$ to 330 $\mathrm{mJ} / \mathrm{cm}^{2}$. While for fluences below $170 \mathrm{~mJ} / \mathrm{cm}^{2}$ the samples were irradiated by the raw beam which was limited to a size of $5 \mathrm{~mm} \times 30 \mathrm{~mm}$ by an aperture, for fluences above $170 \mathrm{~mJ} / \mathrm{cm}^{2}$ a fused silica lens with a focal length of 500 $\mathrm{mm}$ was used to decrease the beam size. Fig. 6 shows the top and cross-sectional views of an untreated sample (a) as well as a series of samples treated with different increasing laser fluences (b) - (d). While laser fluences of $120 \mathrm{~mJ} / \mathrm{cm}^{2}$ and below (not shown here) do not seem to influence the morphology of the layer, fluences of $162 \mathrm{~mJ} / \mathrm{cm}^{2}$ and above lead to melting and significant densification of the particle layer. For a fluence of $245 \mathrm{~mJ} / \mathrm{cm}^{2}$, apart from some holes which might be a result of a not fully homogeneous initial distribution of particles, almost maximal density is reached while grain boundaries between the consolidated particles remain visible. A more detailed description of the shown layers and a comparison of the laser treated layers to conventionally annealed particle layers are given in [19]. An exemplary sample of $\mathrm{ZnO}$ particles on a $\mathrm{SiO}_{2}$ buffer layer can be seen in Fig 7 (a). Experiments with such layer stacks show that the laser fluence needed to achieve a good con- 
solidation of the $\mathrm{ZnO}$ layer are much lower in comparison to the samples without a $\mathrm{SiO}_{2}$ buffer layer as indicated by the simulations. Fig 7 (b) and (c) show such a $\mathrm{ZnO} / \mathrm{SiO}_{2}$ layer stack after irradiation by a laser pulse at a fluence of only $60 \mathrm{~mJ} / \mathrm{cm}^{2}$. The surface and cross-section of the particle layer stack show that a very dense consolidation of the $\mathrm{ZnO}$ layer was achieved while the $\mathrm{SiO}_{2}$ particles appear to be unaffected. The substantial decrease in the laser fluence needed for $\mathrm{ZnO}$ layer consolidation indicates that indeed the applied buffer layer can hamper the conduction of heat from the particle layer towards the substrate resulting in higher layer temperatures and as seen in the simulaton part also increases the time needed for a cool down of the hot $\mathrm{ZnO}$ layer. A longer cool down time could contribute to enable diffusion processes and also to reduce thermal stress in the particle layer.

\section{Conclusion}

In conclusion, our experiments and simulations have shown that excimer laser irradiation is suitable for the consolidation of metal oxide nanoparticle layers. The laser fluences needed for consolidation of $\mathrm{ZnO}$ particle layers could be reduced greatly by adding a buffer layer of $\mathrm{SiO}_{2}$ particles below the $\mathrm{ZnO}$ particle layer. These finding indicate that the transport of heat towards the substrate is significantly hindered by the buffer layer. Simulations of the system showed the same result. At the same time, the temperatures at the border between particle layer stack and substrate remain much lower. Thus, our investigations indicate that such a buffer layer could be used in the low-cost mass production of devices such as solar cells or displays on sensitive substrates like polymers despite the extremely high melting temperature of metal oxide nanoparticles such as ITO or $\mathrm{ZnO}$ while leaving the visual appearance of the layer unchanged. The performance of the buffer layer showed to be highly dependent on its thickness which has to be adapted to the substrate material.

\section{Acknowledgements}

The support of the German Research Foundation (DFG, Graduiertenkolleg 1161/2) is gratefully acknowledged. Additionally, we are grateful for the generous support by Evonik Industries AG. Moreover, the authors gratefully acknowledge funding of the Erlangen Graduate School in Advanced Optical Technologies (SAOT) by the German Research Foundation (DFG) in the framework of the German excellence initiative.

\section{References}

[1] K. Nomura, H. Ohta, A. Takagi, T. Kamiya, M. Hirano, H. Hosono, Nature 432, (2004) 488

[2] J.-S. Lee, M.V. Kovalenko, J. Huang, D.S. Chung, D.V. Talapin, Nat. Nanotechnol. 6, (2011) 348

[3] S. Walther, S. Schäfer, M.P.M. Jank, H. Thiem, W. Peukert, L. Frey, H. Ryssel, Microelectron. Eng. 87, (2010) 2312

[4] A.J. Morfa, G. Beane, B. Mashford, B. Singh, E. D. Gaspera, A. Martucci, P. Mulvaney, J. Phys. Chem. C 114 (2010), 19815
[5] D. Lee, H. Pan, S.H. Ko, H.K. Park, E. Kim, C.P. Grigoropoulos, Appl. Phys. A 107, (2012) 161

[6] M. Baum, I. Alexeev,M. Schmidt, J. Laser Nano/Microeng. 6, (2011) 191

[7] Ü. Özgür, Ya.I. Alivov, C. Liu, A. Teke, M.A. Reshchikov, J. Appl. Phys. 98, (2005) 041301

[8] Y.C. Liu, J.H. Hsieh, S.K. Tung, Thin Solid Films 510, (2006) 32

[9] D.A.G. Bruggeman, Annalen der Physik 5. Folge, 24 (1935) p. 636

[10] H.G. Hirschberg, Handbuch Verfahrenstechnik und Anlagenbau. Chemie, Technik und Wirtschaftlichkeit (Springer, Berlin, 1999) p. 300

[11] O.V. Mazurin, M.V. Streltsina, T.P. Shvaiko

Shvaikovskaya, Handbook of Glass Data (Elsevier, Amsterdam, 1983) p. 55

[12] G.A. Slack, C.J. Glassbrenner, Phys. Rev. 134, (1964) A1058

[13] VDI Gesellschaft, VDI-Wärmeatlas (Springer, Berlin, 2006)

[14] E.D. Palik, Handbook of Optical Constants of Solids (Academic Press, New York, 1985) p. 555

[15] E.N. Bunting, J. Am. Ceram. Soc. 13, (1930) 5

[16] Y. Kinemuchi, M. Mikami, K. Kobayashi, K. Watari, Y. Hotta, J. Electron. Mater. 39, (2010) 2059

[17] M. Takagi, J. Phys. Soc. Jpn. 9 (1954) 359

[18] S. Walther, S. Polster, B. Meyer, M.P.M. Jank, H. Ryssel, L. Frey, J. Vac. Sci. Technol. B 29, (2011) 01A601 [19] M. Baum, S. Polster, M.P.M. Jank, I. Alexeev, L. Frey, M. Schmidt, Appl. Phys. A 107, (2012) 269

(Received: June 13, 2012, Accepted: March 4, 2013) 